\title{
Interpreting spatial terms involves simulating interactions
}

\author{
LAURA A. CARLSON and RYAN KENNY \\ University of Notre Dame, Notre Dame, Indiana
}

\begin{abstract}
Recent research in spatial language has demonstrated that the interpretation of a spatial term depends not only on the geometry of the configuration of the objects being spatially related, but also on extrageometric information, including information about the objects and their interaction. Such effects could emerge from activation of general knowledge of the association between the objects; thus, they should be widely observed. In contrast, they could be more restricted, emerging only in situations in which the spatial language task positions objects in a manner that is consistent with a simulation of their interaction. In two experiments, we test each of these ideas and demonstrate that extrageometric information augments geometric information in the interpretation of spatial terms only when the situation enables the interaction.
\end{abstract}

Spatial descriptions indicate the location of one object (a located object) by spatially relating it to another object (a reference object) whose location is presumed to be known or easily found (Beun \& Cremers, 1998; Levelt, 1996; Talmy, 1983). A traditional view is that the interpretation of such descriptions is based on the geometry of the objects and their configuration (Herskovits, 1986; Landau \& Jackendoff, 1993; Talmy, 1983). For example, in a geometric account, the command Put the mug below the teapot should result in placement of the cup under the center of the teapot. However, research across a variety of spatial terms has demonstrated that extrageometric information, such as knowledge about the objects and their interaction, also has an impact on how spatial terms are interpreted. For example, extrageometric knowledge might cause the listener to place the mug under the spout of the teapot rather than under its center. This has led to the suggestion of a weighted combination of geometric and extrageometric sources (see, e.g., Carlson-Radvansky, Covey, \& Lattanzi, 1999; Coventry \& Garrod, 2004). In the present study, we examine more closely the processes that give rise to extrageometric influence.

\section{Functional Influence: General Knowledge or Simulated Interaction?}

Our test case of an extrageometric influence is the bias to interpret a spatial term relative to a part of a reference object that is functionally important. Carlson-Radvansky et al. (1999) gave participants pictures of reference ob-

A portion of this research was conducted as part of R.K.'s senior honors thesis at the University of Notre Dame. We thank the Undergraduate Research Opportunity Program of the Institute for Scholarship in the Liberal Arts, College of Arts and Letters, University of Notre Dame, for supporting this research. Correspondence concerning this article should be addressed to L. A. Carlson, Department of Psychology, 118-D Haggar Hall, University of Notre Dame, Notre Dame, IN 46556 (e-mail: 1carlson@nd.edu). jects (e.g., a teapot) and pictures of located objects (e.g., a mug) and asked them to place the located objects above or below the reference objects. The reference objects had functional parts that were offset from the center (e.g., the spout of the teapot), enabling an assessment of whether placements were based on geometric features (e.g., the visual center of mass) or on function. For all objects, placements were biased toward the functional part. In the present experiments, we test between two possible accounts of this functional bias: one based on the activation of general knowledge of the objects, and one based on simulation of an interaction between the objects.

General knowledge effect. General knowledge about the objects could result in the functional bias in one of two ways. First, the bias could be due to the functional prominence associated with a particular part of the reference object (e.g., the spout's being critical to the function of the teapot). This account would predict that placements of a located object (regardless of identity) would always be biased toward a particular functional part. Second, the bias could be due to general knowledge of the association between the located object and a functional part, which is consistent with Langacker's (1993) idea of an "active zone" in which certain parts of an object are profiled. This account would predict that placements of located objects would be biased toward different functional parts, with the identity of the located object defining which parts were functionally prominent on the basis of their association (e.g., a mug might highlight the spout of a teapot, whereas a hand might highlight its handle). Such associations are presumably based on co-occurrence information drawn from past experience. Importantly, this account would predict that placements of the located object would always reflect this association and be biased toward the appropriate functional part.

Simulated interaction effect. More restrictively, the simulated interaction account predicts that the functional 
bias effect depends not only on a general association between the objects, but also on whether a simulated interaction among the objects is enabled by the spatial term used to describe their spatial relation. This idea emerges from several recent approaches that link language comprehension to action and perceptual processes (Barsalou, 1999; Coventry \& Garrod, 2004; Glenberg \& Kaschak, 2002; Glenberg \& Robertson, 2000; Zwaan, 2004). In this view, language serves as a cue to the comprehender to construct an experiential simulation that includes perceptual and action components, with details of the situation actively constraining interpretation (see also Chambers, Tanenhaus, Eberhard, Filip, \& Carlson, 2001). According to this account, interpretation of the command Put the mug below the teapot involves construction of a simulation of the interaction between the objects, drawing not only on general knowledge of their association but also on underlying forces and principles that govern their interaction (such as a dynamic-kinematic routines; Coventry \& Garrod, 2004). This simulation must accommodate the spatial term that is specified in the placement instruction, in consistency with the general idea that the simulation is constrained by the demands of the situation (Glenberg \& Kaschak, 2002; Glenberg \& Robertson, 2000). The degree of modification depends on the fit of the definition of the spatial term (functional or geometric) with the interaction. In the case of the teapot and the mug, a functional definition of the spatial term below supports the interaction between the objects, whereas a geometric definition fits less well. Therefore, a bias to place the mug below with respect to the spout should be observed.

In contrast, neither a functional nor a geometric definition of the term above supports the interaction between the objects (i.e., no placement of the mug above the teapot would enable tea to be poured from the pot into the mug). Thus, the interaction is not relevant, and participants should default to applying a geometric definition of above, and therefore show no functional bias. This would be consistent with the more general case of using geometric definitions of spatial terms when spatially relating objects that do not interact (as in The coffee cup is to the left of the telephone).

Note that this account differs from the general knowledge account in predicting that the functional bias will not be observed when the spatial term is inconsistent with the simulated interaction between the objects; in contrast, the general knowledge account predicts that such a bias should always be observed.

\section{EXPERIMENT 1}

To test between the general knowledge and simulated interaction accounts of the functional bias, reference objects were selected that had two distinct functional parts located at opposite ends. For each reference object, two located objects were selected, each interacting with one of the functional parts. An example is a curling iron with the iron tip paired with a wig and the electric cord paired with a power strip (see Figure 1). Table 1 provides a complete list of reference objects, functional parts, and located objects. A neutral located object (beanbag) was also paired with each reference object. Participants placed a given located object (e.g., wig, power strip, beanbag) above, below, or near the reference object.

If the functional bias is due to general knowledge of the functional prominence of a given part of a reference object, then placements should be biased toward the same part of the reference object for all located objects and for all spatial terms (e.g., always toward the iron end of the curling iron). In contrast, if the functional bias is due to general knowledge of the association between a particular part of the reference object and the located object, then placements should be biased toward the relevant functional part as determined by the identity of the located object (e.g., for the wig, biased toward the iron; for the power strip, biased toward the plug). Importantly, this bias should be observed for all spatial terms. Finally, if the functional bias is due to the simulation of an interaction between the objects, then placements should be biased toward the relevant functional parts (as determined by the located objects) only when the constraints imposed by the term are consistent with the interaction.

The simulated interaction and general knowledge accounts can be differentiated when the constraints imposed by the spatial term are inconsistent with the interaction; in these cases, the simulated interaction account predicts that the functional bias should disappear, whereas the general knowledge account predicts that it should remain. For a majority of the stimuli used in the present experiment, the typical direction of interaction is from the side. Thus, the spatial terms above and below restrict placements within a vertical direction that is not consistent with the horizontal direction required for interaction. In contrast, near does not convey a particular direction (Logan \& Sadler, 1996); thus, this term allows placements that are consistent with the interaction. Thus, if simulations of the interaction underlie the functional bias, then placements should be biased toward the relevant functional part for near (e.g., for the wig, placement should be biased toward the curling iron) but not for above and below. In contrast, if knowledge of the association between the located object and the relevant functional part underlies the functional bias, then placements should be biased toward the relevant part for all spatial terms.

\section{Method}

Participants. Seventy-two University of Notre Dame undergraduate students participated in exchange for extra credit for a class. All provided informed consent and were naive with respect to the hypotheses under investigation.

Stimuli. The stimuli were black-and-white versions of color photographs of real objects. Fifteen trios of objects were constructed. Each trio consisted of three located objects and a reference object that had two distinct functional parts located at opposite ends. Two of the located objects each interacted with one functional part of the reference object (see Figure 1 and Table 1). The third (neutral) located object of each trio was one of five different beanbags. We arbitrarily assigned a standard orientation to each reference object, labeling the functional part on the left in this orientation FuncPart 1 and the one on the right FuncPart2. A mirror-image version of each 

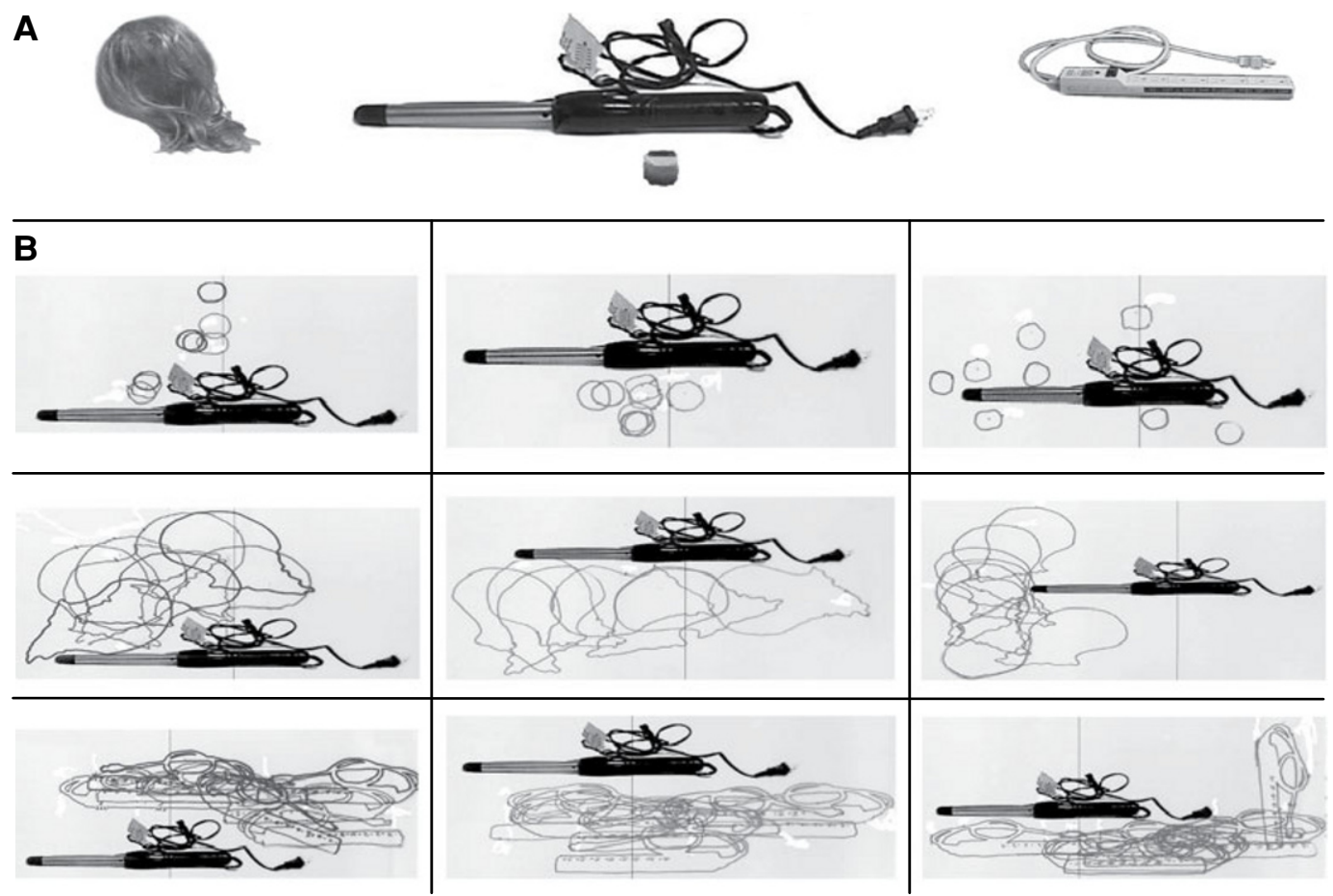

Figure 1. (A) Sample reference object (curling iron), a located object that interacts with the iron tip (wig), a located object that interacts with the electrical cord (power strip), and a neutral object (beanbag) used in Experiment 1. (B) Placements of the beanbag (top row), wig (middle row), and power strip (bottom row) as a function of spatial term (above in column 1, below in column 2, and near in column 3).

object was also constructed to ensure that any biases were not linked to preferences for a particular side. Reference objects were copied onto the center of an $8.5 \times 11$-in. sheet of paper (in landscape orientation) with ample white space (55-119 $\mathrm{mm}$ to the left and right, $60-100 \mathrm{~mm}$ at the top and bottom). Located objects were cut out along their edges with no surrounding space.

Procedure. On a given trial, the reference object was placed in front of the participant and identified (e.g., "This is a curling iron."). A located object (coated on the back with adhesive) was also identified (e.g., "This is a wig.") and then handed to the participant with the instruction to "Put the [located object] above/below/near the [reference object]." The participant placed the located object at any orientation in any location, adjusting as desired. Each participant received each reference object once, yielding 15 trials, with 7 (or 8) of the trials with FuncPart1 on the left and 8 (or 7) with FuncPart1 on the right (counterbalanced across participants). Each participant performed 5 trials for each spatial term and 5 for each type of located object; these factors were fully crossed by item across but not within participants.

\section{Results and Discussion}

All placements for a given object were traced onto a transparency containing the reference object in its standard orientation. Placements were combined across the

Table 1

Trios of Objects Used in the Placement Task

\begin{tabular}{lllllr}
\hline & & & & & Horizontal \\
Reference Object & FuncPart1 & Located Object 1 & FuncPart2 & Located Object 2 & Extent (mm) \\
\hline Curling iron & iron tip & wig & plug & power strip & 158 \\
Trumpet & horn & mute & mouth & mouthpiece & 120 \\
Dump truck & loader & marbles & bed & logs & 118 \\
Leash & clip end & collar & handle & hook & 156 \\
Mousetrap game & diving board & man & cheese & cage & 164 \\
Flashlight & light end & lens & battery end & battery & 122 \\
Syringe & needle end & needle & medicine end & stopper & 92 \\
Compact & eye shadow & applicator & blush & brush & 113 \\
Lock & metal loop & chain & mechanism & keys & thread \\
Needle & point & pin cushion & eye & nipple & 108 \\
Baby bottle brush & wide bristles & bottle & small bristles & beer bottle & 152 \\
Bottle opener & corkscrew & wine bottle & pry tab & film & 150 \\
Camera & lens & lens cap & base & CD player & 37 \\
Headphones & earpiece & head & jack & base & 162 \\
Microphone stand & mike holder & microphone & base end & 128 \\
\hline
\end{tabular}


standard and nonstandard orientations by drawing on both sides of the transparency. Placements for a representative object are shown in Figure 1B.

For each placement of each reference object, the midpoint of the located object, based on its horizontal extent (as positioned by the participant), was measured (in $\mathrm{mm}$ ) relative to a line running through the center of the reference object (in $\mathrm{mm}$ ), with deviations toward FuncPart1 coded as negative and deviations toward FuncPart 2 coded as positive. Given that different objects had different horizontal extents (see Table 1), deviations were converted into percentages of the distance between the midpoint of the reference object and the respective part, with $0 \%$ indicating no bias (i.e., a strictly geometric placement) and $\pm 100 \%$ indicating complete bias (i.e., a strictly functional placement).

Mean percent deviations as a function of the spatial term and located object are provided in Table 2. Deviations were submitted to a 3 (located object: related to FuncPart1 vs. related to FuncPart 2 vs. neutral) $\times 3$ (spatial term: above vs. below vs. near) repeated measures ANOVA with objects as the independent variable. ${ }^{1}$ There was a main effect of located object $\left[F(2,28)=15.2, M S_{\mathrm{e}}=.194\right]$, no main effect of spatial term $(F<1)$, and a significant interaction between located object and spatial term $[F(4,56)=8.4$, $\left.M S_{\mathrm{e}}=.096\right]$. In general, the deviations for the FuncPart1 object were toward FuncPart1 (as indicated by the negative sign), whereas the deviations for the FuncPart 2 object were toward FuncPart2 (as indicated by the positive sign). Indeed, for all terms there was a significant difference between the FuncPart1 and FuncPart2 deviations [all $t \mathrm{~s}(14)>2.4, p \mathrm{~s}<.028$ ], indicating that the part deemed functional depended on the identity of the located object. The interaction was due to this effect's being very strong for near, with deviations significantly different from 0 $[t(14)>2.8, p<.012]$, but weak for above and below, with deviations not significantly different from 0 [both $t \mathrm{~s}(14)<1.7, p \mathrm{~s}>.11]$. Finally, there was a significant deviation for the beanbag for near but not for above or below, perhaps indicating an underlying prominence for FuncPart1.

These data rule out the general knowledge account, according to which the functional bias is due to the functional prominence of a given part, since placements were not systematically biased toward only one functional part. They also rule out the general knowledge account, accord-

Table 2

Mean Percent Deviations as a Function of Spatial Term and Type of Located Object in Experiment 1

\begin{tabular}{cccc}
\hline \multicolumn{4}{c}{ and Type of Located Object in Experiment 1 } \\
\cline { 2 - 4 } & \multicolumn{3}{c}{ Located Object } \\
\cline { 2 - 4 } Splated to & Related to & Neutral \\
\hline Above & FuncPart1 & FuncPart2 & -7.5 \\
Below & -10.6 & 11.2 & 0.3 \\
Near & -21.1 & 8.1 & $-26.3^{*}$ \\
\hline
\end{tabular}

Note-Within each term, differences between FuncPart1 and FuncPart2 were significant at $p<.03$. *Significantly different from $0(p<.05)$. ing to which the functional bias is due to the association between the objects; such association was held constant across spatial terms, but a strong bias was observed only for near. This pattern supports the simulated interaction account, by which the interaction between the objects defines the part deemed functional, with term constraints dictating whether or not such functional information impacts placements.

\section{EXPERIMENT 2}

In Experiment 2, we sought further evidence for the simulated interaction account, focusing on four critical points. First, in Experiment 1 we failed to find a strong functional bias with the spatial terms above and below; in contrast, Carlson-Radvansky et al. (1999) observed strong biases with these terms with their set of stimuli. We attribute this difference to the fact that the objects used by Carlson-Radvansky et al. typically interacted from above and below and were therefore consistent with the directional restrictions imposed by these terms. In contrast, in our Experiment 1 the typical direction of interaction for most objects was from the side and, consequently, inconsistent with the directional constraints imposed by the terms above and below. In Experiment 2, we offer a direct test of this idea by using a picture of a reference object (a shape sorter) with one functional part for which the interaction is from above (the plus-sign slot) and another for which the interaction is from the right (the moon slot), and pictures of located objects that interact with the above part (plus sign) and the right part (moon) of the reference object. These objects are shown in Figure 2. Placements were made for the spatial terms above, near, and right. If the match between the direction of the interaction and the direction imposed by the spatial term is critical, then for the plus sign, only above and near placements should show a functional bias; for the moon, only right and near placements should show a functional bias. Such predicted placements are designated as functional in Figure 2. Moreover, such placements should be made in such a way that the interaction could be fulfilled.

Second, placements of objects for which the spatial term is inconsistent with the interaction allow a further test between the general knowledge account and the simulated interaction account. Specifically, for right placements of the plus sign, if general knowledge (GK) of the association of the objects is important independently of the spatial term, then placements should be as close as possible to the plus-sign slot. These placements could cluster in one of two locations, depending on whether the participants defined the spatial term with respect to the shape sorter as a whole object (designated $G K$-whole object-right) or with respect to the plus-sign slot (designated $G K$-part-right); however, importantly, such placements in either cluster would not be positioned in a manner to fulfill the interaction. Finally, a geometric placement would be midway down the right side of the object (designated geometric-right in Figure 2). Similarly, 


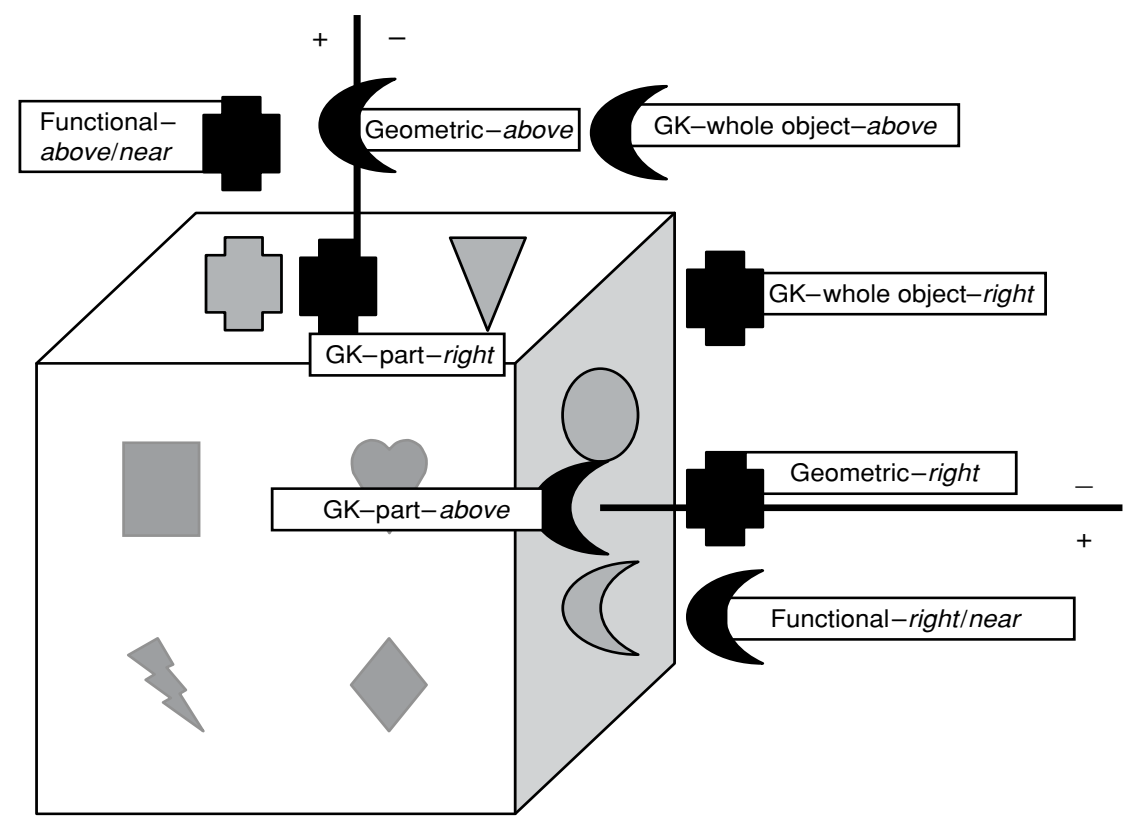

Figure 2. Predicted placements of the moon and plus sign around the shape sorter in Experiment 2. Vertical and horizontal axes are the reference lines running through the middle of the top and right faces, respectively, of the shape sorter. Deviations were measured from the midpoints, with " + " indicating the direction toward the functional parts and "-" indicating the direction away from the functional parts (see text for details).

for above placements of the moon, general knowledge placements would be as close to the moon slot as possible, clustering in a location based on defining the spatial term either with respect to the shape sorter as a whole object (designated GK-whole object-above) or with respect to the moon slot (designated GK-part-above). Again, such placements would not be positioned to interact. Geometric placements would be centered above the top surface (designated geometric-above).

Third, Coventry and Garrod (2004) have pointed out that, in addition to geometry and object knowledge, dynamic-kinematic routines corresponding to forces or principles that govern the objects' interaction may contribute to the Carlson-Radvansky et al. (1999) functional bias. With respect to the shape sorter, a pertinent dynamickinematic routine would capture the sense in which the shape must be positioned over the respective hole in order to successfully drop into the sorter. Coventry and Garrod suggest that dynamic-kinematic routines influence the interpretation of projective terms such as above and right, but not proximity terms such as near. Thus, the shape sorter offers an interesting test of whether the influence of a given dynamic-kinematic routine will change as a function of the type of spatial term (projective vs. proximity).

Finally, in Experiment 1 a variety of real objects was used, with the consequences that the reference objects and the located objects all varied in size and shape, the degree of association among the objects was not equated, and it is possible that not all the objects interacted in the same manner. To rule out the potential influence of these factors, in Experiment 2 we focused on a single reference object and two located objects, matched in size, that were equally associated with the reference object and that interacted with it in the same manner, thereby eliciting the same dynamic-kinematic routine.

\section{Method}

Participants. Seventy-two University of Notre Dame undergraduate students participated. All provided informed consent and were naive with respect to the hypotheses under investigation. After completing the shape-sorter trial, the participants completed an unrelated experiment.

Stimuli and Procedure. Each participant completed one trial, with 12 participants assigned to each spatial term (above, right, or near) and located object (moon or plus sign) combination. The participants were tested individually. They were shown the picture of the shape sorter and told, "Here is a picture of a shape sorter." They were then handed a cutout shape (the located object) and told, "Here is a shape." Note that the shape was not further identified as a moon or a plus sign because we did not want to bias the participants toward an interpretation that would focus them on the corresponding functional slot. Finally, the participants were asked to "Place the shape above/to the right of/near the shape sorter." The shape sorter is drawn to scale in Figure 2. The front face measured $105 \mathrm{~mm}$ on each side, the top face measured $105 \mathrm{~mm}$ across from left to right and $35 \mathrm{~mm}$ from top to bottom, and the right face measured $35 \mathrm{~mm}$ across from left to right and $105 \mathrm{~mm}$ from top to bottom. Each located object fit within a $16-\mathrm{mm}^{2}$ bounding box. The slots were equally spaced across the top, front, and right faces of the sorter. The center of the plus-sign slot was $25 \mathrm{~mm}$ from the center of the top face (indicated by the vertical axis), and the center of the moon slot was $25 \mathrm{~mm}$ from the center of the right face (indicated by the horizontal axis). 


\section{Results and Discussion}

Placements for all the participants are shown in Figure 3. Deviations were coded relative to either a horizontal axis (for placements to the right) or a vertical axis (for placements above the shape sorter). Deviations toward the two functional parts (moon slot and plus-sign slot) were coded as positive, and those away from the functional parts were coded as negative (see Figure 2). Deviations were converted into percentages of the distance between the axis and the center of the functional part, and are presented in Table 3 as a function of located object and spatial term. A 2 (located object) $\times 3$ (term) between-subjects ANOVA revealed a main effect of term $[F(2,66)=16.1$, $\left.M S_{\mathrm{e}}=2,852\right]$ and no effect of located object $(F<1)$, suggesting that the objects were equally associated with their functional parts, and a significant interaction between located object and term $\left[F(2,66)=7.03, M S_{\mathrm{e}}=2,852\right]$.

Plus-sign placements. Placements for above and near clustered near the plus-sign slot, indicating a functional bias; thus, sign is positive and differs significantly from 0 , which serves as an index of a geometric placement. Note also that a majority of these placements ( 9 of 12 for near and 10 of 12 for above) were positioned to fulfill the interaction. However, for right-a term inconsistent with the interaction between the plus sign and the plus-sign slot - there were two clusters of placements. One cluster of 8 placements was geometric, centered on the right side, and another cluster of 4 placements was placed to the right of the shape sorter's plus-sign slot, although not in a position to interact with it. Whether the deviation is averaged across these two sets of placements or restricted to the right placements on the right side of the reference object, the sign of the deviation is negative and significantly smaller than those for above and near.

Moon placements. Placements for near and right clustered mostly near the moon slot, indicating a functional bias - the sign is positive and significantly differs from 0 . A majority of these placements were positioned so as to fulfill the interaction (conservatively, 9 of 12 for near and 7 of 12 for right). However, for above there were three clusters of placements. One set (4 placements) was centered by the top face, in keeping with a geometric prediction; one set (2 placements) was placed above the moon slot on the right face, although not in a position to interact with it. A third set (6 placements) was placed directly on the moon slot, in a position to functionally interact with it. Note that for these placements to be acceptable for the term above, the participants would be required to base their definition of above on an intrinsic reference frame based on the object rather than on an environmental reference frame. When deviations were averaged across these sets, the above placements were significantly less functional than the near placements; deviations averaged only for the placements environmentally above the sorter were negative and significantly different from those for near and for right.

Comparison across terms. When the located object was placed in a manner consistent with its interaction with the reference object, there was no significant difference in the deviations for near versus above for plus sign placements $(p=.21)$ or for near versus right for moon placements $(p=.16)$. In fact, the direction in the means is opposite what one might expect if proximity terms were influenced only by functional information but projective terms were influenced by both functional and dynamic-kinematic routines. This suggests that projective and proximity terms may be similarly influenced by functional and dynamic-kinematic routines.

In summary, the critical finding of Experiment 2 was that the functional bias was observed for both located objects when the spatial term enabled the interaction between the located object and the reference object. However, when the term constraints were inconsistent with the

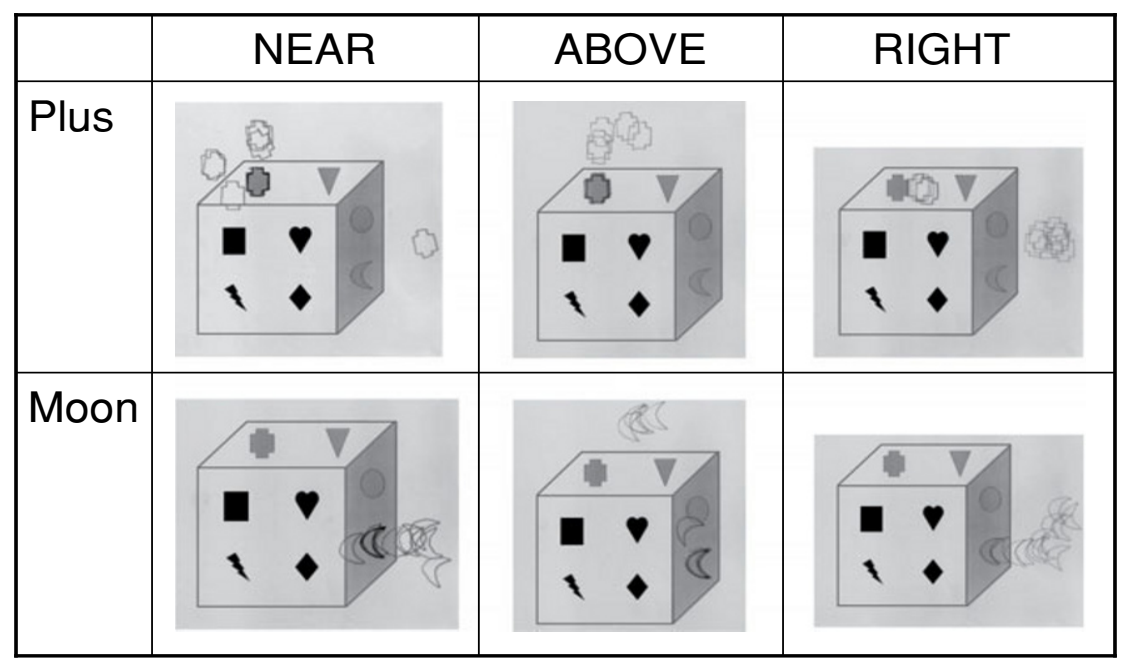

Figure 3. Placements of the plus sign (top row) and moon (bottom row) as a function of spatial terms (near, above, and right) in Experiment 2. 
Table 3

Mean Percent Deviations as a Function of Spatial Term and Located Object in Experiment 2 for All Placements, Restricted to Placements of Right Relative to the Horizontal Axis, and Restricted to Placements of Above Relative to the Vertical Axis

\begin{tabular}{|c|c|c|c|c|}
\hline \multirow[b]{2}{*}{ Spatial Term } & \multicolumn{2}{|c|}{$\begin{array}{c}\text { Plus Sign } \\
\text { (Slot Above) }\end{array}$} & \multicolumn{2}{|c|}{$\begin{array}{c}\text { Moon } \\
\text { (Slot on Right) }\end{array}$} \\
\hline & All & Restricted & All & Restricted \\
\hline Above & $83.0^{\mathrm{a}^{*}}$ & & $40.0^{\mathrm{d} \dagger}$ & $-45.0^{f \dagger}$ \\
\hline Right & $-9.7^{b}$ & $-28.0^{\mathrm{c}^{*}}$ & $56.7^{\mathrm{d}^{*}}$ & \\
\hline Near & $121.3^{\mathrm{a}^{*}}$ & & $100.3 \mathrm{e}^{*}$ & \\
\hline
\end{tabular}

Note-Superscripts a, b, and c correspond to the plus-sign data, with each letter indicating a different significant difference among conditions; superscripts d, e, and f correspond to the moon data, with each letter indicating a different significant difference among conditions. The restricted placements exclude four right placements of the plus sign that were by the plus-sign slot on the top face of the shape sorter, with right defined relative to the plus-sign slot rather than the shape sorter; and eight above placements of the moon that were by the moon slot on the right face of the shape sorter, with above defined relative to the moon slot rather than the shape-sorter. * ${ }^{*}$ Significantly different from $0(p<$ .05). †Significantly different from $0(p<.09)$.

interaction, placements were based either on a geometric definition of the spatial term or on a general, noninteractive association between the objects.

\section{GENERAL DISCUSSION}

Interpretation of spatial descriptions of the location of one object with respect to another object depends not only on the geometric configuration of the objects but also on extrageometric information, including object knowledge and dynamic-kinematic information about their interaction. The present article demonstrates that such extrageometric information has an effect on the interpretation of a spatial term when term constraints are compatible with a simulated interaction between the objects.

By simulated interaction, we mean the construction of a situation model of the described event that integrates general knowledge of the association among the objects, dynamic-kinematic information that may define the interaction, and constraints imposed by the spatial term, with this information represented as a set of perceptual and action representations (Zwaan, 2004; see also Barsalou, 1999; Coventry \& Garrod, 2004; Glenberg \& Kaschak, 2002). Thus, when the term constraints are consistent with the interaction of the objects, the simulation incorporates a functional definition of the spatial term; when the term constraints are inconsistent with the interaction, the simulation draws on a geometric definition of the spatial term. Within the domain of spatial language, this idea is similar to Coventry and Garrod's conceptualization of a weighted combination of various types of information (geometric, dynamic-kinematic, and object knowledge) during the online interpretation of a spatial description. However, one implication of the present study is that it may not be possible to classify a given set of terms as more or less geometric, functional, or dependent upon dynamic-kinematic routines; rather, understanding the relative contributions of geometric and extrageometric factors will necessarily depend on an assessment of the situation in which the spatial terms are being interpreted that is consistent with an embodied perspective of language processing.

\section{REFERENCES}

Barsalou, L. W. (1999). Perceptual symbol systems. Behavioral \& Brain Sciences, 22, 577-660.

Beun, R.-J., \& Cremers, A. H. M. (1998). Object reference in a shared domain of conversation. Pragmatics \& Cognition, 6, 121-152.

Carlson-Radvansky, L. A., Covey, E. S., \& Lattanzi, K. M. (1999). "What" effects on "where": Functional influences on spatial relations. Psychological Science, 10, 516-521.

Chambers, C. G., Tanenhaus, M. K., Eberhard, K. M., Filip, H., \& CARlson, G. N. (2001). Circumscribing referential domains during real-time language comprehension. Journal of Memory \& Language, 47, 30-49.

Coventry, K. R., \& Garrod, S. C. (2004). Saying, seeing and acting: The psychological semantics of spatial prepositions. Hove, U.K.: Psychology Press.

Glenberg, A. M., \& KaschaK, M. P. (2002). Grounding language in action. Psychonomic Bulletin \& Review, 9, 558-565.

Glenberg, A. M., \& Robertson, D. A. (2000). Symbol grounding and meaning: A comparison of high-dimensional and embodied theories of meaning. Journal of Memory \& Language, 43, 379-401.

Herskovits, A. (1986). Language and spatial cognition: An interdisciplinary study of the prepositions in English. Cambridge: Cambridge University Press.

LANDAU, B., \& JACKendoff, R. (1993). "What" and "where" in spatial language and spatial cognition. Behavioral \& Brain Sciences, 16, 217-265.

LANGACKER, R. W. (1993). Grammatical traces of some "invisible" semantic constructs. Language Sciences, 15, 323-355.

Levelt, W. J. M. (1996). Perspective taking and ellipsis in spatial descriptions. In P. Bloom, M. A. Peterson, L. Nadel, \& M. Garrett (Eds.), Language and space (pp. 77-108). Cambridge, MA: MIT Press.

Logan, G. D., \& SADLER, D. (1996). A computational analysis of the apprehension of spatial relations. In P. Bloom, M. A. Peterson, L. Nadel, \& M. Garrett (Eds.), Language and space (pp. 494-529). Cambridge, MA: MIT Press.

TALMY, L. (1983). How language structures space. In H. L. Pick \& L. P. Acredolo (Eds.), Spatial orientation: Theory, research, and application (pp. 225-282). New York: Plenum.

ZwaAn, R. A. (2004). The immersed experiencer: Toward an embodied theory of language comprehension. In B. H. Ross (Ed.), The psychology of learning and motivation: Advances in research and theory (Vol. 44, pp. 35-62). San Diego: Academic Press.

\section{NOTE}

1. We were not able to conduct comparable analyses using participants as the random factor because spatial term and located object were not fully crossed within participants. However, each participant made five placements for each spatial term. Collapsing across the objects used for these placements revealed a main effect of term $[F(2,142)=4.6]$, with near deviations $(M=-11.7)$ significantly greater than above $(M=$ $-1.8)$ and below $(M=-1.4)$ deviations $(p \mathrm{~s}<.03)$, which did not differ from each other. Each participant also made five placements with each object. Collapsing across the terms used for these placements resulted in a main effect of object $[F(2,142)=24.8]$, with the deviations for the FuncPart1 object $(M=-18.9$, biased toward FuncPart1) significantly different from the deviations for the FuncPart2 object $(M=12.7$, biased toward FuncPart2) and with both differing from the beanbag's deviations $(M=-7.4)($ all $p \mathrm{~s}<.02)$. These data are consistent with the items analyses.

(Manuscript received November 3, 2004; revision accepted for publication November 24,2005 .) 